\title{
Patient and provider perspectives on self-administered electronic substance use and mental health screening in HIV primary care
}

\author{
Alexandra N. Lea ${ }^{1 *} \mathbb{D}$, Andrea Altschuler ${ }^{1}$, Amy S. Leibowitz ${ }^{1}$, Tory Levine-Hall' ${ }^{1}$ Jennifer McNeely², \\ Michael J. Silverberg ${ }^{1}$ and Derek D. Satre ${ }^{1,3}$
}

\begin{abstract}
Background: Substance use disorders, depression and anxiety disproportionately affect people with HIV (PWH) and lead to increased morbidity and mortality. Routine screening can help address these problems but is underutilized. This study sought to describe patient and provider perspectives on the acceptability and usefulness of systematic electronic, self-administered screening for tobacco, alcohol, other substance use, and mental health symptoms among patients in HIV primary care.
\end{abstract}

Methods: Screening used validated instruments delivered pre-appointment by both secure messaging and clinicbased tablets, with results integrated into the electronic health record (EHR). Qualitative analysis of semi-structured interviews with 9 HIV primary care providers and 12 patients in the 3 largest HIV primary care clinics in the Kaiser Permanente Northern California health system who participated in a clinical trial evaluating computerized screening and behavioral interventions was conducted. Interviews were audio-recorded and transcribed. A thematic approach was utilized for coding and analysis of interview data using a combination of deductive and inductive methods.

Results: Four key themes were identified: (1) perceived clinical benefit of systematic, electronic screening and EHR integration for providers and patients; (2) usefulness of having multiple methods of questionnaire completion; (3) importance of the patient-provider relationship to facilitate completion and accurate reporting; and (4) barriers, include privacy and confidentiality concerns about reporting sensitive information, particularly about substance use, and potential burden from repeated screenings.

Conclusions: Findings suggest that electronic, self-administered substance use and mental health screening is acceptable to patients and may have clinical utility to providers. While offering different methods of screening completion can capture a wider range of patients, a strong patient-provider relationship is a key factor in overcoming barriers and ensuring accurate patient responses. Further investigation into facilitators, barriers, and utility of electronic screening for PWH and other high-priority patient populations is indicated.

Trial registration ClinicalTrials.gov, NCT03217058. Registered 13 July 2017, https://clinicaltrials.gov/ct2/show/NCT03 217058

Keywords: Screening, Smoking, Alcohol, Depression, Anxiety, HIV

*Correspondence: Alexandra.N.Lea@kp.org

1 Division of Research, Kaiser Permanente Northern California, 2000 Broadway, Oakland, CA 94612, USA

Full list of author information is available at the end of the article

\section{Background}

People with HIV (PWH) have higher rates of mental health and substance use disorders (SUD) than the general population, and these conditions are associated with original author(s) and the source, provide a link to the Creative Commons licence, and indicate if changes were made. The images or other third party material in this article are included in the article's Creative Commons licence, unless indicated otherwise in a credit line to the material. If material is not included in the article's Creative Commons licence and your intended use is not permitted by statutory regulation or exceeds the permitted use, you will need to obtain permission directly from the copyright holder. To view a copy of this licence, visit http://creativecommons.org/licenses/by/4.0/. The Creative Commons Public Domain Dedication waiver (http://creativeco mmons.org/publicdomain/zero/1.0/) applies to the data made available in this article, unless otherwise stated in a credit line to the data. 
poorer HIV outcomes and increased mortality [1]. SUDs, including alcohol, opioid and stimulant use disorders [2-5], and depression are 2-4 times more prevalent in PWH compared to people without HIV [6, 7]. Anxiety is also common but has been under investigated relative to depression $[8,9]$.

Routine screening is essential for identifying these comorbidities [10-13], but is often underutilized due to lack of resources, time constraints, and stigma [14, 15]. When screening does occur, there is variability in question content, frequency, and documentation by providers $[16,17]$, and patients often underreport symptoms, particularly alcohol and other drug use problems $[15,18]$.

Patient self-report can be influenced by a variety of factors, including mode of question administration and sensitivity of content $[18,21]$. Compared with face-toface interviews, self-administered, computerized measures can facilitate more accurate reporting of stigmatized behavior [19-21], improve fidelity [14, 22, 23] and increase patient comfort [24]. On the other hand, stigma, perceived judgment, negative experiences with providers or confidentiality breaches can be barriers to disclosure of anxiety and depression [25-28] as well as substance use [29]. A trusting relationship between patient and provider can be an important facilitator to disclosure in a primary care setting and help address these barriers [23, 30, 31].

The current study aimed to investigate these issues in the context of a large screening and intervention trial focused on self-administered, computerized substance use and mental health screening in HIV primary care. This paper reports on the qualitative findings from interviews with primary care providers and patients to identify common perspectives on screening practices, as well as facilitators and barriers to accurate patient reporting in an HIV primary care setting.

\section{Methods}

The Promoting Access to Care Engagement (PACE) trial examines a novel approach to screening, which combined substance use and mental health measures in a single, self-report questionnaire for PWH systematically administered every 6 months, coinciding with primary care appointments [32]. The PACE study was designed to evaluate the implementation, effectiveness, and cost of routine, electronic screening and brief behavioral treatments for SUDs, depression and anxiety. The qualitative data reported here focused on screening rather than subsequent interventions delivered by behavioral health specialists. The study occurred in the 3 largest HIV primary care clinics (Oakland, Sacramento, and San Francisco) in Kaiser Permanente Northern California (KPNC), which collectively serve over 5000 PWH. Patients were asked to complete 2 validated, self-administered screening measures prior to a scheduled appointment: The Tobacco, Alcohol, Prescription medication, and other Substance use (TAPS) instrument [33], which has been shown to have good sensitivity and specificity for identifying problem use in the general primary care population, particularly for tobacco, alcohol, and marijuana; and the Adult Outcomes Questionnaire (AOQ), which includes the Patient Health Questionnaire (PHQ-9) for depression [34] and the Generalized Anxiety Disorder (GAD-2) for anxiety [35], both of which have also been shown to have high levels of sensitivity and specificity in the same population. These were combined in a single questionnaire (the TAPS/AOQ).

KPNC offers patients an online portal that allows access to appointments, lab results, other health care information and secure messaging with their providers. Patients with access to the portal were invited via secure message to complete the TAPS/AOQ online prior to their visit, and those who did not were invited to complete it on a tablet upon arrival in the clinic. TAPS/AOQ responses were automatically recorded in the patients' electronic health record (EHR) and were visible to providers and behavioral health specialists embedded in HIV primary care (Sacramento) or general primary care (Oakland and San Francisco). Key clinical trial outcomes include screening completion rates; utilization of specialty addiction and mental health treatment; and HIV viral control based on EHR data [32]. Outcome analyses are in process.

The focus of analysis here are qualitative telephone interviews conducted with providers and patients from participating clinics to better understand barriers and facilitators associated with implementation and clinical utility of substance use and mental health screening. Verbal informed consent and permission for recording and transcription were obtained. Participants in the provider interviews did not receive compensation. Participants in the patient interviews received a $\$ 50$ gift card after the interview. Study procedures were approved by the KPNC and University of California, San Francisco Institutional Review Boards.

\section{Participants}

Interviewees were providers and adult patients from each of the three participating clinics. Patient interviewees who previously completed the TAPS/AOQ were selected via convenience sampling by participating clinicians. The study team partnered with providers to identify participants for recruitment from all 3 facilities with varying degrees of self-reported substance use and mental health symptoms. Eligible patients received a study information sheet from their provider either at their in-person clinic 
visit or via secure message after a virtual visit. Interested patients allowed their provider to give their contact information to study staff, who completed the telephone interview. We provide descriptive characteristics of participants, including demographics (age, sex, race, and HIV risk group) and scores on the TAPS, GAD-2, and PHQ-9, and compare these to the overall sample of PACE patients from all three study clinics.

\section{Interview guides}

Provider interviews included questions about substance use and mental health screening practices pre-trial; experience with the TAPS/AOQ, including facilitators and barriers to implementation; perceived success of implementation; feedback from patients regarding the screening; and value and feasibility of utilizing the TAPS/AOQ as part of clinic operations after study conclusion. See Additional file 1: Appendix S1 for the provider interview guide. Patient interviews included questions about experiences with substance use and mental health screening prior to TAPS/AOQ implementation; experiences completing the questionnaire; facilitators and barriers to completion; and any interactions with providers based on screening results. See Additional file 2: Appendix S2 for the patient interview guide.

\section{Data collection}

Provider and patient interviews were conducted by two investigators (DDS and MJS) and a Masters level team member (ANL). Clinician interviews lasted 30-45 min and patient interviews lasted up to $30 \mathrm{~min}$ over a 9-month period. Interviews were audio recorded and transcribed verbatim. Interviews were conducted until study investigators believed thematic saturation was reached [36, 37] based on the concepts of data adequacy [38-40] and data source triangulation [41].

\section{Qualitative analysis}

We used a thematic approach, combining deductive and inductive reasoning, for coding and analysis [42, 43]. This approach was chosen to identify and evaluate both explicit and implicit perspectives provided by interviewees. With input from the research team, author AA developed separate codebooks for the provider and patient interviews based on interview guides and field notes. Four authors (AA, DDS, ANL and ASL) independently coded a quarter of the transcripts in each category. Differences in coding were resolved via consensus, and final codebooks were established. Data analysis was managed using NVivo statistical software version 12 (QSR International) and followed standard methods for qualitative research to ensure analysis was systematic and verifiable $[44,45]$.

\section{Results}

Nine providers and 12 patients participated in interviews from all three sites. Providers ( 8 physicians and one nurse practitioner) included 6 men and 3 women. Interview participant characteristics and those of patients in the overall PACE cohort are included in Table 1. The interview and intervention samples had similar demographics (age [median $=59$ vs. 55], gender [92\% male vs. $92 \%$ male], risk group [ $83 \% \mathrm{MSM}$ vs. $76 \% \mathrm{MSM}$ ], and race/ ethnicity [50\% White; $17 \%$ Black; 33\% Hispanic vs. 53\% White; 18\% Black; 16\% Hispanic]). Findings show that while demographic characteristics and HIV risk group were similar in the interview sample, interviewees scored higher on measures indicative of substance use disorder risk, anxiety and depression.

Key themes identified from patient and provider interviews are described below. Additional illustrative quotes, by theme, are shown in Table 2 .

\section{Theme 1: Clinical benefits and value of systematic screening}

Patients reported that prior to implementation of the TAPS/AOQ during the trial, substance use screening focused mainly on tobacco and alcohol. Mental health screening was "more vague or ambiguous, like thow are you doing?'; 'how's everything going?"' All patients

Table 1 Characteristics of patient interview cohort vs. overall PACE cohort

\begin{tabular}{|c|c|c|c|c|}
\hline \multirow[t]{2}{*}{ Total patients } & \multicolumn{2}{|c|}{ Pt. interview cohort } & \multicolumn{2}{|c|}{ PACE cohort } \\
\hline & 12 & $100 \%$ & 2892 & $100 \%$ \\
\hline \multicolumn{5}{|c|}{ Substance use (high/med.) } \\
\hline Any risk & 10 & $83 \%$ & 1759 & $61 \%$ \\
\hline \multicolumn{5}{|l|}{ Depression/anxiety } \\
\hline PHQ9 ( $\geq 10)$ & 4 & $33 \%$ & 381 & $13 \%$ \\
\hline GAD2 $(\geq 3)$ & 3 & $25 \%$ & 378 & $13 \%$ \\
\hline \multicolumn{5}{|l|}{ Demographics } \\
\hline Age, median (IQR) & 59 & $(41-61)$ & 55 & $(46-62)$ \\
\hline \multicolumn{5}{|l|}{ Sex, $n(\%)$} \\
\hline Male & 11 & $92 \%$ & 2656 & $92 \%$ \\
\hline Female & 1 & $8 \%$ & 236 & $8 \%$ \\
\hline \multicolumn{5}{|l|}{ Risk group, $n(\%)$} \\
\hline MSM & 10 & $83 \%$ & 2189 & $76 \%$ \\
\hline Heterosexual & 2 & $17 \%$ & 346 & $12 \%$ \\
\hline IV drug use & 0 & $0 \%$ & 187 & $6 \%$ \\
\hline Other & 0 & $0 \%$ & 170 & $6 \%$ \\
\hline \multicolumn{5}{|l|}{ Race, $n(\%)$} \\
\hline White & 6 & $50 \%$ & 1532 & $53 \%$ \\
\hline Black & 2 & $17 \%$ & 535 & $18 \%$ \\
\hline Hispanic & 4 & $33 \%$ & 474 & $16 \%$ \\
\hline Other & 0 & $0 \%$ & 351 & $12 \%$ \\
\hline
\end{tabular}




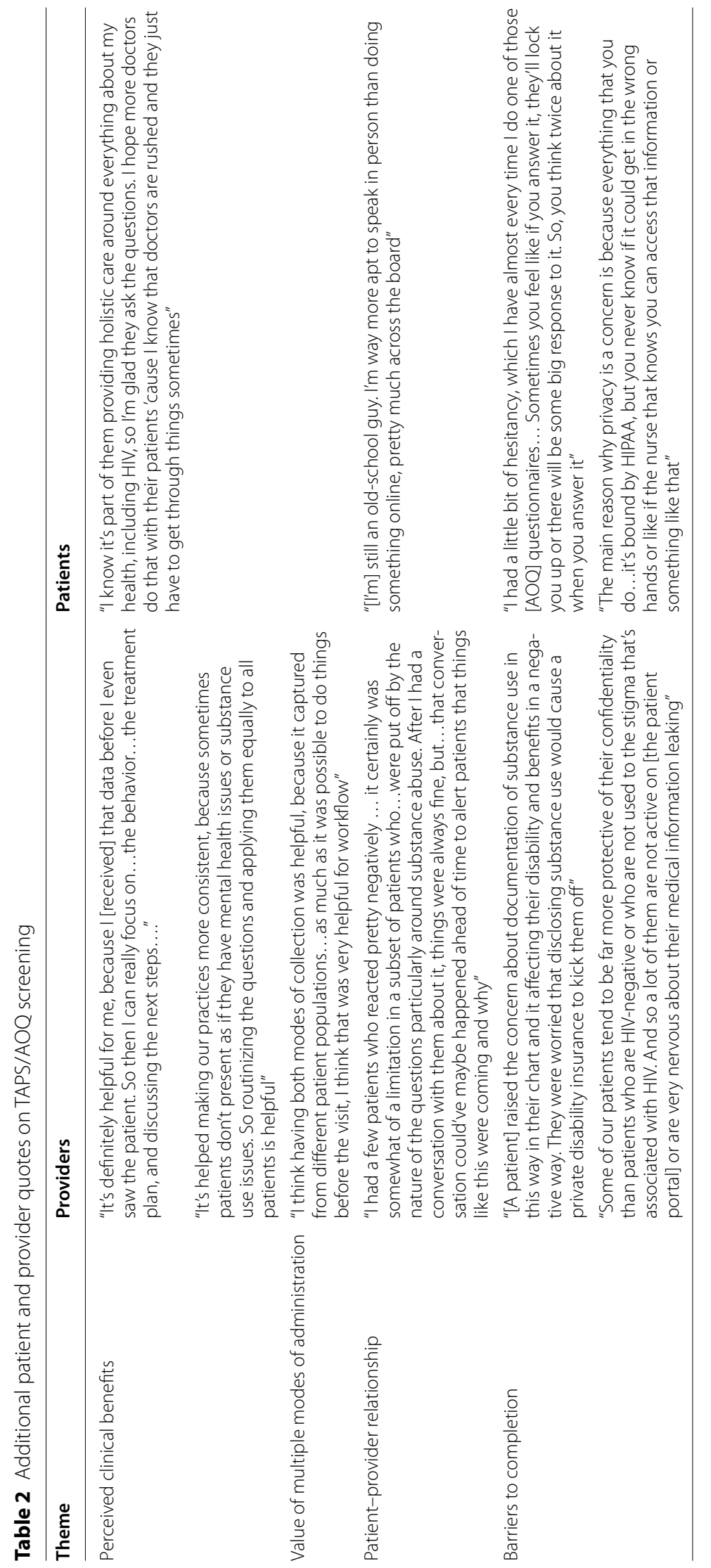


supported the new screening questions, with one patient indicating "not only is it [the] physical aspect [of HIV] that you're dealing with, but it's also an emotional and mental thing because it's a really big lifestyle change... It's good to know where a person's mental health lies, and then, shortly after, it became like a substance abuse thing...So, I think it's appropriate to ask those types of questions."

Providers reported that the TAPS/AOQ reinforced the importance of screening and increased their awareness of the prevalence of substance use and mental health issues for PWH. Most providers also felt that the TAPS/ AOQ identified more patients with these issues, particularly those that were less severe, and whose conditions they were previously unaware of. One provider noted, "The nice thing with [the TAPS/AOQ] is it certainly has picked up things that I haven't asked about, and I can think of people that throw in... party drugs, ecstasy, that I may not have picked up on or they may not have told me. But it comes out in those questionnaires."

Providers reported different practices regarding frequency of mental health and substance use screening before the PACE trial, often dependent on whether a given patient was known to have existing problems. Providers differed in where they would document such issues in the EHR. Only tobacco and alcohol screening were regularly completed in a uniform manner [46, 47]. Although providers utilized some validated instruments such as the PHQ-9 prior to TAPS/AOQ implementation, mental health screening practices varied by clinician. Providers found that consistent screening practices and standardized documentation of results helped inform clinical decisions. One said, "I would share results with the patients in terms of their TAPS/AOQ responses and we could follow them together to look for improvements or areas of lack of improvement and perhaps worsening over time."

Inclusion of screening for substances other than alcohol and tobacco meant that providers gained more information about their patients' use, while self-administration preserved limited appointment time for other patient needs. One noted: "It's really helpful, because... it saves appointment time in order to have that information there....You're walking into a situation where you've done that screen, you know how much time you're going to prioritize to that."

\section{Theme 2: Value of different screening modalities}

All patients reported it was easy to complete the TAPS/ $\mathrm{AOQ}$, regardless of self-administration method (patient portal or in-clinic tablet). They also reported preferring different methods to access the TAPS/AOQ depending on how and when they access care. Some were familiar with the portal and regularly utilized it to interact with providers, and therefore preferred the convenience of online completion, while others stated that they were more likely to complete the questionnaire in the clinic, when they felt they were in a "patient mindset." Some indicated that tablet completion helped them transition from hectic daily routines to focusing on their healthcare upon arrival in the clinic. One patient described the difference as: "I did it on my computer, and that takes like pre-preparation in order to actually pre-access it. And, once I'm actually present within the [clinic] building itself, I guess it's more so trying to be present and prepare myself for my doctor's appointment."

Providers felt that having online and tablet options for TAPS/AOQ completion captured different patient populations and both were valuable. Online completions were convenient for patients who preferred to complete the questionnaires in advance and eased the administrative burden on clinic workflows. Tablet completions were valuable for those who lacked internet access or were not registered to use the portal: "We certainly don't want to miss those folks, because in some ways they may be even a higher risk patient population."

\section{Theme 3: Impact of patient-provider communication and relationship on screening}

The quality of patient-provider communication and relationships were important factors in patients' level of comfort disclosing substance use and mental health issues. Patients appreciated when providers mentioned their TAPS/AOQ results during their visit, whether there were issues or not, and said such discussions made it more likely for them to disclose issues in the future. One patient commented about the value of their provider having this information: "[The provider mentioning TAPS/ AOQ results] makes me feel good because then it feels like he's actually paying attention to what I put time on.... I felt like he cared about what I was going through, and that made me feel good."

Patients also reported that developing a relationship with their provider over time and discussing questions and responses in person made them more likely to report substance use and mental health issues. One stated: "I want that personal connection.... I feel fortunate having these long-term relationships with the doctors that I can say anything and feel comfortable saying anything that I want." Patients and providers both reported that availability of TAPS/AOQ screening results informed patient-provider discussions and proved an important opportunity to strengthen and improve the relationship. Even for patients who chose not to complete the screening, receipt of the questionnaire could still prompt a 
conversation regarding why the questions were being asked and/or why it was not completed.

Both patients and providers discussed two key aspects of the secure message: (1) sent from the patient's provider, versus from a general health system email address, and (2) included an explanation regarding the importance of screening completion. One provider stated: "I think people, when they get emails, you know, 'from your doctor,' they think it really comes from your doctor, that your doctor sits down and writes this and sends it to you, without recognizing that 90 percent of those, your doctor doesn't even know get sent. So, they're like, 'Wow. Why are you asking me this?' Not like, 'Why is [the health system] doing this?' It was very personal." A patient noted: "I'm glad that it was explained to me what it was all about and why I was being asked those questions, and once I was told, it was like, 'Oh, okay. No problem. I understand that now.".

\section{Theme 4: Barriers to completion—privacy/confidentiality and questionnaire fatigue}

The most frequently mentioned barriers to TAPS/AOQ completion were concerns regarding privacy and confidentiality. Patients were concerned that their answers might be used in a negative or discriminatory manner related to their care, employment or benefits. One patient stated: "I mean, everything is a part of your medical record. So, I feel that you always kind of have to be wary of how in depth you go with everything." Some patients were also concerned with the sensitivity of the TAPS/ AOQ content and their responses being misunderstood because the questionnaire does not capture the nuances of substance use, recovery, and mental health. For example, several reported having to answer affirmatively to TAPS/AOQ questions due to the questionnaire's wording, but not having the opportunity to contextualize their answers (e.g., previous substance use and mental health issues resolved many years prior).

Providers also reported that, while many PWH were familiar with substance use and mental health screening, some had concerns regarding confidentiality, and preferred that providers document substance use and mental health responses in their clinical notes rather than as answers to screening tools. They felt that patient privacy was more protected in a free text clinical note versus structured EHR data that is more readily retrievable, including by health plan administrators, other clinicians and staff. One provider noted: "Some patients have had issues with disclosure around substance use. And primarily they will be untruthful on the questionnaire because it doesn't come from me directly. And they will say, 'I actually use, X, Y, Z, but I didn't report it here,' and primarily for confidentiality and employment reasons, they don't want that in their medical record-even if it gets noted in my note, it's different than if they just kind of answered on the questionnaire."

Finally, another common barrier to completion was a sense of burden or fatigue due to past receipt of other health surveys. The KPNC healthcare system regularly utilizes online questionnaires in many departments for patients who use the patient portal. Patients sometimes reported feeling overwhelmed by the number of questionnaires they were asked to complete, which could lead to them not completing any of those requested. Furthermore, many providers expressed concern about patients being asked to complete the TAPS/AOQ on a regular basis, especially in cases where there was an established patient-provider relationship and no previous history of symptoms. For example, one stated: "[One patient] really [took] offense, and that was basically conveying... 'You know me. Why would you ask me these questions? You know I don't smoke. You know I don't drink. You know I have never used drugs.' The same provider stated: "I am concerned that patients that score very low on all of these measures, when queried again in 6 months, might get some fatigue around it and feel frustrated."

\section{Discussion}

This qualitative study examined patient and provider perspectives on the implementation of systematic, selfadministered substance use and mental health screening in three large HIV primary care clinics in an integrated health care system. We identified 4 themes among patients and providers regarding their experiences utilizing the TAPS/AOQ tool: (1) perceived clinical benefits; (2) value of multiple modes of administration; (3) importance of the patient-provider relationship as a facilitator to substance use or mental health disclosure; and (4) privacy/confidentiality concerns and questionnaire fatigue as primary barriers to screening completion.

Our findings suggest that, although substance use and mental health screening was occurring prior to TAPS/ AOQ implementation, screening practices were highly variable, which is consistent with previous reports of substance use and mental health screening in similar settings $[16,17]$. As noted above, standard KPNC practice already routinely screened all primary care patients for alcohol and tobacco use [46, 47], but the TAPS/AOQ enabled screening for tobacco, alcohol, and all major drug classes in a single instrument. Given the recently revised US Preventive Services Task Force recommendation that routine drug screening should be part of highquality primary care for all adults [48], this aspect of the TAPS/AOQ makes it well suited to meet provider needs, particularly in HIV primary care. Providers reported the benefit of consistent screening practices for all 
substances, standardized documentation of results, and periodic rescreening and that TAPS/AOQ implementation increased their awareness of patients' substance use and mental health problems, enabled them to track risk levels and symptoms over time, and often enhanced patient/provider relationships. Of note, providers found value in the long-term monitoring of screening results for patients who initially reported symptoms, both for clinical decision making and strengthening the patientprovider relationship through informed discussions. Although the AOQ depression and anxiety measures have been validated for monitoring over time, the TAPS and other substance use screening tools have not.

All patients indicated that the self-administered screening was highly acceptable, consistent with previous findings [33, 49]. Prior research has provided evidence for patient acceptability of a self-administered TAPS format $[33,49,50]$, while our results build on those findings to show the utility of multiple modes of self-administration (online patient portal and in-clinic tablet) in meeting the needs of different patient populations. Both patients and providers felt that having both options available allowed for more flexibility in administration and an increased likelihood of completion. This flexibility may be especially important to capture younger populations who regularly utilize technology to access care, as well as safety net populations who may not have internet or smartphone access.

Previous research has also indicated that a good provider-patient relationship, while important in all medical care, is particularly valuable for patients with substance use and mental health concerns, where having established trust with a provider can reduce feelings of shame around disclosure [23, 30, 31, 51]. Our results further showed that the most important facilitator for completion of electronic screening was a strong provider-patient relationship in which feelings of trust have been established. Patients and providers felt that discussing TAPS/AOQ responses led to strengthened relationships by increasing feelings of trust and patient comfort with provider, which led to more accurate responses from patients. For those who completed the TAPS/AOQ online, receiving it directly from their provider and including an explanation of its importance were key factors in their decision to complete the screening.

Finally, PWH may place greater weight on confidentiality and privacy [25-28], which is reflected in reports that the sensitivity of drug use and mental health information was a common barrier. Some patients reported hesitation to complete the screening because they worried that their responses, if formally recorded in their EHR, might negatively impact their care, benefits, or employment; consistent with previous findings [30, 52]. Although patients were not formally asked if perceived benefits of screening outweighed risks, the overall positive comments suggest that they thought screening was valuable and, despite the inclusion of sensitive topics, content areas were relevant to their care. A strong patient-provider relationship characterized by open discussion and lack of judgment, in which the provider can explain and emphasize the importance of capturing these results on an ongoing basis, can allay these fears and increase the likelihood of accurate self-report. Patients may also suffer from questionnaire fatigue over time, potentially impacting satisfaction with care and decreasing the quality of the screening [53-57]. Careful consideration of administration schedule is warranted, including mechanisms to vary frequency based on patterns of patient responses, e.g., fewer screenings for those who consistently report minimal substance use or mental health symptoms.

\section{Study limitations}

There were several limitations to this study. A small sample was interviewed, patient participants were primarily male (although this is representative of the population of PWH in KPNC), and the study was based in a private health care system. Convenience sampling by providers for patient interviews may have resulted in limiting participants to those who had a more positive experience with the clinic or the study in general. Additionally, although demographic characteristics of the interview sample were very similar to that of the overall PACE cohort, interviewees scored higher on measures indicative of substance use disorder risk, anxiety and depression. Interviewee perspectives may be less representative of the clinic population, which includes patients with lower or no risk. However, given the intent of the study to evaluate screening and treatment for these conditions, we believe that perspectives of people with higher-severity problems were important to include. Given the barriers noted, such as privacy concerns and questionnaire fatigue, future implementation efforts with screening systems such as this must carefully address these challenges. For example, assessing responses after multiple administrations could provide insight on how regular screening may specifically influence responses and relationships with providers over time, particularly for those who report few or no substance use problems or mental health symptoms.

\section{Conclusions}

This study evaluated patient and primary care provider perspectives regarding newly implemented self-reported substance use and mental health screening practices in HIV primary care. The use of the self-administered electronic screening tool proved 
successful in providing systematic screening and documentation of results. Both modalities of completion (online vs. tablet) as well as the tool itself had high levels of patient acceptability, and self-administration both preserved appointment time for providers to address patient care needs as well as captured substance use and mental health issues not previously reported. Regardless of mode of self-administration, a strong provider-patient relationship appears to be the most important factor in screening completion and accuracy, helping to mitigate patient privacy concerns. Regular screening and review of results between providers and patients can serve as an opportunity to strengthen this relationship and improve substance use and mental health interventions in primary care.

\section{Abbreviations}

AOQ: Adult Outcomes Questionnaire; EHR: Electronic health record; GAD-2: Generalized Anxiety Disorder; KPNC: Kaiser Permanente Northern California; PACE: Promoting Access to Care Engagement; PHQ-9: Patient Health Questionnaire; PWH: People with HIV; SUD: Substance use disorder; TAPS: The Tobacco, Alcohol, Prescription medication and other Substance use Instrument.

\section{Supplementary Information}

The online version contains supplementary material available at https://doi. org/10.1186/s13722-022-00293-7.

Additional file 1: Appendix S1. Provider Interview Guide. Qualitative interview guide used to complete interviews with providers.

Additional file 2: Appendix S2. Patient Interview Guide. Qualitative interview guide used to complete interviews with patients.

\section{Acknowledgements}

We wish to thank Sujaya Parthasarathy for assistance in study design, and Hannah Jang, Courtney Ellis and Gina Smith-Anderson for assistance in project management. We thank Gary Zin, Nancy Facher, and Nicholas Shapiro at The Permanente Medical Group Consulting Services for technical assistance; Drs. Sally Slome, Jason Flamm, and C. Bradley Hare and the clinic staff at the Kaiser Permanente primary care HIV clinics in Oakland, Sacramento, and San Francisco.

\section{Authors' contributions}

ANL planned, recruited informants, conducted interviews, coded and analyzed data, wrote and revised manuscript. AA conducted interviews, coded and analyzed data, and revised manuscript. ASL conducted interviews, coded and analyzed data, and revised manuscript. TLH, analyzed data. JM revised manuscript. MJS planned, secured funding, conducted interviews, and revised manuscript. DDS planned, secured funding, conducted interviews, coded data, and revised manuscript. All authors read and approved the final manuscript.

\section{Funding}

This study was supported by the National Institute on Drug Abuse (R01 DA043139) and the National Institute on Alcohol Abuse and Alcoholism (K24 AA025703).

\section{Availability of data and materials}

The qualitative data analyzed is not publicly available to protect participant identities. Anonymized copies of interview transcripts are available from the corresponding author on reasonable request. Copies of the study interview guides as well as the screening instrument are available upon request.

\section{Declarations}

Ethics approval and consent to participate

This study has been approved by the Kaiser Permanente Northern California and University of California, San Francisco Institutional Review Boards. The participants were informed of the study's purpose and methods, and that their participation would be confidential and voluntary.

\section{Consent for publication}

Not applicable.

\section{Competing interests}

The authors declare no competing interests.

\section{Author details}

${ }^{1}$ Division of Research, Kaiser Permanente Northern California, 2000 Broadway, Oakland, CA 94612, USA. ${ }^{2}$ Department of Population Health, Section on Tobacco, Alcohol, and Drug Use, New York University Grossman School of Medicine, 180 Madison Ave., New York, NY 10016, USA. ${ }^{3}$ Department of Psychiatry and Behavioral Sciences, Weill Institute for Neurosciences, University of California, San Francisco, 401 Parnassus Avenue, Box 0984, San Francisco, CA 94143, USA.

Received: 14 July 2021 Accepted: 26 January 2022

Published online: 09 February 2022

References

1. DeLorenze GN, Satre DD, Tsai A, Quesenberry CP, Weisner C. Mortality following diagnosis of psychiatric disorders and co-occurring substance use disorders among HIV-infected patients. AIDS Patient Care STDS. 2010;24(11):705-12

2. Chander G, Josephs J, Fleishman JA, et al. Alcohol use among HIVinfected persons in care: results of a multi-site survey. HIV Med. 2008;9(4):196-202.

3. Hartzler B, Dombrowski JC, Crane HM, et al. Prevalence and predictors of substance use disorders among HIV care enrollees in the United States. AIDS Behav. 2017:21(4):1138-48.

4. Maeda JL, Lee KM, Horberg M. Comparative health systems research among Kaiser Permanente and other integrated delivery systems: a systematic literature review. Perm J. 2014;18(3):66-77.

5. Mimiaga MJ, Reisner SL, Grasso C, et al. Substance use among HIVinfected patients engaged in primary care in the United States: findings from the centers for AIDS research network of integrated clinical systems cohort. Am J Public Health. 2013;103(8):1457-67.

6. Bing EG, Burnam MA, Longshore D, et al. Psychiatric disorders and drug use among human immunodeficiency virus-infected adults in the United States. Arch Gen Psychiatry. 2001;58(8):721-8.

7. Schumacher JE, McCullumsmith C, Mugavero MJ, et al. Routine depression screening in an HIV clinic cohort identifies patients with complex psychiatric co-morbidities who show significant response to treatment. AIDS Behav. 2013;17(8):2781-91.

8. Brandt C, Zvolensky MJ, Woods SP, Gonzalez A, Safren SA, O'Cleirigh CM. Anxiety symptoms and disorders among adults living with HIV and AIDS: a critical review and integrative synthesis of the empirical literature. Clin Psychol Rev. 2017:51:164-84.

9. Heywood W, Lyons A. HIV and elevated mental health problems: diagnostic, treatment, and risk patterns for symptoms of depression, anxiety, and stress in a national community-based cohort of gay men living with HIV. AIDS Behav. 2016;20(8):1632-45.

10. Substance Abuse and Mental Health Services Administration and Health Resources and Services Administration. The case for behavioral health screening in HIV care settings. HHS publication no. SMA-16-4999. Rockville: Substance Abuse and Mental Health Services Administration; 2016.

11. Chichetto NE, Mannes ZL, Allen MK, Cook RL, Ennis N. HIV care provider perceptions and approaches to managing unhealthy alcohol use in primary HIV care settings: a qualitative study. Addict Sci Clin Pract. 2019;14(1):21. 
12. Gaines MT, Duke CC, Henny KD. Mental health screening practices among primary care providers in high HIV burden areas of the south: does having patients with HIV matter? J Behav Health Serv Res. 2021;48(1):103-11.

13. Shacham E, Nurutdinova D, Satyanarayana V, Stamm K, Overton ET. Routine screening for depression: identifying a challenge for successful HIV care. AIDS Patient Care STDS. 2009;23(11):949-55.

14. Bradley KA, Lapham GT, Hawkins EJ, et al. Quality concerns with routine alcohol screening in VA clinical settings. J Gen Intern Med. 2011;26(3):299-306.

15. Hormes JM, Gerhardstein KR, Griffin PT. Under-reporting of alcohol and substance use versus other psychiatric symptoms in individuals living with HIV. AIDS Care. 2012;24(4):420-3.

16. Campbell ANC, Wolff M, Weaver $L$, Jarlais DD, Tross S. "It's never just about the HIV": HIV primary care providers' perception of substance use in the era of "universal." Antiretrovir MedTreat AIDS Behav. 2018;22(3):1006-17.

17. Ridgway JP, Uvin A, Schmitt J, et al. Natural language processing of clinical notes to identify mental illness and substance use among people living with HIV: retrospective cohort study. JMIR Med Inform. 2021;9(3): e23456.

18. Roux P, Cohen J, Lascoux-Combe C, et al. Determinants of the underreporting of alcohol consumption by HIV/HCV co-infected patients during face-to-face medical interviews: the role of the physician. Drug Alcohol Depend. 2011;116(1-3):228-32.

19. Satre DD, Wolfe W, Eisendrath S, Weisner C. Computerized screening for alcohol and drug use among adults seeking outpatient psychiatric services. Psychiatr Serv. 2008;59(4):441-4.

20. Wight RG, Rotheram-Borus MJ, Klosinski L, Ramos B, Calabro M, Smith R. Screening for transmission behaviors among HIV-infected adults. AIDS Educ Prev. 2000;12(5):431-41.

21. McNeely J, Adam A, Rotrosen J, et al. Comparison of methods for alcohol and drug screening in primary care clinics. JAMA Netw Open. 2021;4(5): e2110721.

22. Williams E, Achtmeyer CE, Rittmueller SE, Lapham GT, Chavez LJ, Thomas RM, Berger DB, Bradley KA. Factors underlying quality problems with alcohol screening in routine care. Addict Sci Clin Pract. 2013;8:A85.

23. Williams EC, Achtmeyer CE, Thomas RM, et al. Factors underlying quality problems with alcohol screening prompted by a clinical reminder in primary care: a multi-site qualitative study. J Gen Intern Med. 2015;30(8):1125-32.

24. Spear SE, Shedlin M, Gilberti B, Fiellin M, McNeely J. Feasibility and acceptability of an audio computer-assisted self-interview version of the alcohol, smoking and substance involvement screening test (ASSIST) in primary care patients. Subst Abus. 2016;37(2):299-305.

25. Conway FN, Rountree MA, Jones KV. Serving the co-morbid mental health and substance use needs of people with HIV. Community Ment Health J. 2021:57:1328-39.

26. Lee RS, Kochman A, Sikkema KJ. Internalized stigma among people living with HIV-AIDS. AIDS Behav. 2002;6(4):309-19.

27. Peltzer K, Ramlagan S. Perceived stigma among patients receiving antiretroviral therapy: a prospective study in KwaZulu-Natal, South Africa. AIDS Care. 2011;23(1):60-8.

28. Rueda S, Gibson K, Rourke SB, Bekele T, Gardner S, Cairney J. Mastery moderates the negative effect of stigma on depressive symptoms in people living with HIV. AIDS Behav. 2012;16(3):690-9.

29. Pinto RM, Chen Y, Park SE. A client-centered relational framework on barriers to the integration of HIV and substance use services: a systematic review. Harm Reduct J. 2019;16(1):71.

30. McNeely J, Kumar PC, Rieckmann T, et al. Barriers and facilitators affecting the implementation of substance use screening in primary care clinics: a qualitative study of patients, providers, and staff. Addict Sci Clin Pract. 2018;13(1):8.

31. Parker D, Byng R, Dickens C, McCabe R. Patients' experiences of seeking help for emotional concerns in primary care: doctor as drug, detective and collaborator. BMC Fam Pract. 2020;21(1):35.

32. Satre DD, Anderson AN, Leibowitz AS, et al. Implementing electronic substance use disorder and depression and anxiety screening and behavioral interventions in primary care clinics serving people with
HIV: protocol for the promoting access to care engagement (PACE) trial. Contemp Clin Trials. 2019;84: 105833.

33. McNeely J, Wu LT, Subramaniam G, et al. Performance of the tobacco, alcohol, prescription medication, and other substance use (TAPS) tool for substance use screening in primary care patients. Ann Intern Med. 2016;165(10):690-9.

34. Kroenke K, Spitzer RL, Williams JB. The PHQ-9: validity of a brief depression severity measure. J Gen Intern Med. 2001;16(9):606-13.

35. Plummer F, Manea $L$, Trepel D, McMillan D. Screening for anxiety disorders with the GAD-7 and GAD-2: a systematic review and diagnostic metaanalysis. Gen Hosp Psychiatry. 2016;39:24-31.

36. Saunders B, Sim J, Kingstone T, et al. Saturation in qualitative research: exploring its conceptualization and operationalization. Qual Quant. 2018;52(4):1893-907.

37. Vasileiou K, Barnett J, Thorpe S, Young T. Characterising and justifying sample size sufficiency in interview-based studies: systematic analysis of qualitative health research over a 15-year period. BMC Med Res Methodol. 2018;18(1):148.

38. Erickson F, Wittrock MC. Handbook of research on teaching. Washington, D.C: American Educational Research Association; 1986. p. 119-61.

39. Levitt HM, Motulsky SL, Wertz FJ, Morrow SL, Ponterotto JG. Recommendations for designing and reviewing qualitative research in psychology: promoting methodological integrity. Qual Psychol. 2017:4(1):2-22.

40. Morrow SL. Quality and trustworthiness in qualitative research in counseling psychology. J Couns Psychol. 2005;52(2):250-60.

41. Carter N, Bryant-Lukosius D, DiCenso A, Blythe J, Neville AJ. The use of triangulation in qualitative research. Oncol Nurs Forum. 2014;41(5):545-7.

42. Braun V, Clarke V. Using thematic analysis in psychology. Qual Res Psychol. 2006;3(2):77-101.

43. Luborsky M. The identification and analysis of themes and patterns. In: Gubrium JF, Sankar A, editors. Qualitative methods in aging research. New York: Sage; 1994. p. 189-210.

44. O'Brien BC, Harris IB, Beckman TJ, Reed DA, Cook DA. Standards for reporting qualitative research: a synthesis of recommendations. Acad Med. 2014;89(9):1245-51.

45. Tong A, Sainsbury P, Craig J. Consolidated criteria for reporting qualitative research (COREQ): a 32-item checklist for interviews and focus groups. Int J Qual Health Care. 2007;19(6):349-57.

46. Lam JO, Levine-Hall T, Hood N, et al. Smoking and cessation treatment among persons with and without HIV in a US integrated health system. Drug Alcohol Depend. 2020;213: 108128.

47. Mertens JR, Chi FW, Weisner CM, et al. Physician versus non-physician delivery of alcohol screening, brief intervention and referral to treatment in adult primary care: the ADVISe cluster randomized controlled implementation trial. Addict Sci Clin Pract. 2015;10:26.

48. Krist AH, Davidson KW, Mangione CM, et al. Screening for unhealthy drug use: US preventive services task force recommendation statement. JAMA. 2020;323(22):2301-9.

49. Adam A, Schwartz RP, Wu LT, et al. Electronic self-administered screening for substance use in adult primary care patients: feasibility and acceptability of the tobacco, alcohol, prescription medication, and other substance use (myTAPS) screening tool. Addict Sci Clin Pract. 2019:14(1):39.

50. McCormick KA, Cochran NE, Back AL, Merrill JO, Williams EC, Bradley KA How primary care providers talk to patients about alcohol: a qualitative study. J Gen Intern Med. 2006;21(9):966-72.

51. Rahm AK, Boggs JM, Martin C, et al. Facilitators and barriers to implementing screening, brief intervention, and referral to treatment (SBIRT) in primary care in integrated health care settings. Subst Abus. 2015;36(3):281-8.

52. Hamilton LWS, Wilens T, Kannry J, Rosenthal RN, Goldfeld K, et al. Patient attitudes toward substance use screening and discussion in primary care. Addict Sci Clin Pract. 2020;15(Suppl 2):A103.

53. Fischer F, Kleen S. Possibilities, problems, and perspectives of data collection by mobile apps in longitudinal epidemiological studies: scoping review. J Med Internet Res. 2021;23(1): e17691.

54. Meirte J, Hellemans N, Anthonissen M, et al. Benefits and disadvantages of electronic patient-reported outcome measures: systematic review. JMIR Perioper Med. 2020;3(1): e15588. 
55. Porter SR, Whitcomb ME, Weitzer WH. Multiple surveys of students and survey fatigue. New Dir Inst Res. 2004;2004(121):63-73.

56. Saunders EC, Moore SK, Gardner T, et al. Screening for substance use in rural primary care: a qualitative study of providers and patients. J Gen Intern Med. 2019;34(12):2824-32.

57. Lapham GT, Rubinsky AD, Williams EC, et al. Decreasing sensitivity of clinical alcohol screening with the AUDIT-C after repeated negative screens in VA clinics. Drug Alcohol Depend. 2014;142:209-15.

\section{Publisher's Note}

Springer Nature remains neutral with regard to jurisdictional claims in published maps and institutional affiliations.

- fast, convenient online submission

- thorough peer review by experienced researchers in your field

- rapid publication on acceptance

- support for research data, including large and complex data types

- gold Open Access which fosters wider collaboration and increased citations

- maximum visibility for your research: over $100 \mathrm{M}$ website views per year

At BMC, research is always in progress.

Learn more biomedcentral.com/submissions 